PROCEEDINGS OF THE

AMERICAN MATHEMATICAL SOCIETY

Volume 127, Number 9, Pages 2529-2533

S 0002-9939(99)05414-3

Article electronically published on May 19, 1999

\title{
ON THE SEMISIMPLICITY OF PURE SHEAVES
}

\author{
LEI FU
}

(Communicated by Ron Donagi)

\begin{abstract}
We obtain a criteria for a pure sheaf to be semisimple. As a corollary, we prove the following: Let $X_{0}$ and $S_{0}$ be two schemes over a finite field $\mathbf{F}_{q}$, and let $f_{0}: X_{0} \rightarrow S_{0}$ be a proper smooth morphism. Assume $S_{0}$ is normal and geometrically connected, and assume there exists a closed point $s$ in $S_{0}$ such that the Frobenius automorphism $F_{s}$ acts semisimply on $H^{i}\left(X_{\bar{s}}, \overline{\mathbf{Q}_{l}}\right)$, where $X_{\bar{s}}$ is the geometric fiber of $f_{0}$ at $s$ (this last assumption is unnecessary if the semisimplicity conjecture is true). Then $R^{i} f_{0 *} \overline{\mathbf{Q}_{l}}$ is a semisimple sheaf on $S_{0}$. This verifies a conjecture of Grothendieck and Serre provided the semisimplicity conjecture holds. As an application, we prove that the galois representations of function fields associated to the $l$-adic cohomologies of $K 3$ surfaces are semisimple. We also get a theorem of Zarhin about the semisimplicity of the Galois representations of function fields arising from abelian varieties.
\end{abstract}

The proof relies heavily on Deligne's work on Weil conjectures.

\section{INTRODUCTION}

Let $\mathbf{F}_{q}$ be a finite field with $q$ elements. Choose an algebraic closure $\mathbf{F}$ of $\mathbf{F}_{q}$. Throughout this paper, schemes, morphisms and sheaves defined on the base field $\mathbf{F}_{q}$ are denoted by letters with subscripts 0 and we indicate the base extension from $\mathbf{F}_{q}$ to $\mathbf{F}$ by dropping the subscripts 0 . Schemes and morphisms are separated and of finite type.

Let $X_{0}$ be a scheme over $\mathbf{F}_{q}$, let $\mathcal{F}_{0}$ be a constructible $\overline{\mathbf{Q}_{l}}$-sheaf on $X_{0}$, and let $\iota: \overline{\mathbf{Q}_{l}} \rightarrow \mathbf{C}$ be an isomorphism. Recall that $\mathcal{F}_{0}$ is called $\iota$-pure with weight $w$ if for every closed point $x$ of $X_{0}$ and for every eigenvalue $\lambda$ of the (geometric) Frobenius automorphism $F_{x}$ on $\mathcal{F}_{\bar{x}}$, the absolute value of $\iota(\lambda)$ is $N(x)^{w / 2}$, where $N(x)$ is the number of elements of the residue field $k(x)$. Also recall that giving a lisse $\overline{\mathbf{Q}_{l}}$-sheaf on a connected scheme is the same as giving a $\overline{\mathbf{Q}_{l}}$-representation of the fundamental group of the scheme. So we can talk about the irreducibility and semisimplicity of a lisse sheaf.

If $X_{0}$ is a normal geometrically connected scheme over $\mathbf{F}_{q}$ and if $\mathcal{F}_{0}$ is a $\iota$ pure lisse $\overline{\mathbf{Q}_{l}}$-sheaf on $X_{0}$, then by a theorem of Deligne ([D1], 3.4.1 (iii)), $\mathcal{F}$ is a semisimple sheaf on $X$. In this paper, based on Deligne's work, we prove the following:

Theorem. Let $X_{0}$ be a normal geometrically connected scheme of finite type over $\mathbf{F}_{q}$ and let $\mathcal{F}_{0}$ be a $\iota$-pure lisse $\overline{\mathbf{Q}_{l}}$-sheaf on $X_{0}$. If there exists a closed point $x$ of

Received by the editors August 11, 1997.

1991 Mathematics Subject Classification. Primary 14F20, 14G15.

(C)1999 American Mathematical Society 
$X_{0}$ such that the Frobenius automorphism $F_{x}$ on $\mathcal{F}_{\bar{x}}$ is semisimple, then $\mathcal{F}_{0}$ is a semisimple sheaf on $X_{0}$.

Here a linear transformation on a vector space is said to be semisimple if the corresponding matrix is diagonalizable.

Corollary. Let $X_{0}$ and $S_{0}$ be two schemes over the finite field $\mathbf{F}_{q}$, and let $f_{0}$ : $X_{0} \rightarrow S_{0}$ be a proper smooth morphism. Assume $S_{0}$ is normal and geometrically connected, and assume there exists a closed point $s$ in $S_{0}$ such that the Frobenius automorphism $F_{s}$ acts semisimply on $H^{i}\left(X_{\bar{s}}, \overline{\mathbf{Q}_{l}}\right)$, where $X_{\bar{s}}=X_{0} \otimes_{k(s)} \overline{k(s)}$ is the geometric fiber of $f_{0}$ at $\bar{s}$. Then $R^{i} f_{0 *} \overline{\mathbf{Q}}_{l}$ is a semisimple sheaf on $S_{0}$.

Proof. By the proper and smooth base change theorem, we know $R^{i} f_{0 *} \overline{\mathbf{Q}_{l}}$ is lisse. By Deligne's theorem (i.e. Weil's conjecture), $R^{i} f_{0 *} \overline{\mathbf{Q}_{l}}$ is pure. By assumption, $F_{s}$ acts semisimply on $\left(R^{i} f_{*} \overline{\mathbf{Q}_{l}}\right)_{\bar{s}}=H^{i}\left(X_{\bar{s}}, \overline{\mathbf{Q}_{l}}\right)$. So by the theorem, $R^{i} f_{0 *} \overline{\mathbf{Q}_{l}}$ is a semisimple sheaf on $S_{0}$.

The semisimplicity conjecture states that for any proper smooth scheme $X_{0}$ over $\mathbf{F}_{q}$, the geometric Frobenius correspondence $F$ acts semisimply on $H^{i}\left(X, \overline{\mathbf{Q}_{l}}\right)$. If this conjecture is true, then in the above corollary, we don't need the assumption that $F_{s}$ acts semisimply on $H^{i}\left(X_{\bar{s}}, \overline{\mathbf{Q}_{l}}\right)$. So provided the semisimplicity conjecture holds, the above corollary verifies for schemes over finite field a conjecture of Serre and Grothendieck stated in $[\mathrm{T}]$.

The semisimplicity conjecture is proved to be true for the following varieties over finite field:

(a) smooth projective curves ([W]),

(b) abelian varieties ([W]),

(c) $K 3$ surfaces ([D2], [PS]).

Denote the function field of $S_{0}$ by $K$. Let $Y$ be a smooth projective variety defined over $K$. Assume $Y$ is in one of the following families:

(a) smooth projective curves,

(b) abelian varieties,

(c) $K 3$ surfaces.

Then by the above corollary the Galois representation

$$
\operatorname{Gal}(\bar{K} / K) \rightarrow H^{i}\left(Y \otimes_{K} \bar{K}, \overline{\mathbf{Q}_{l}}\right)
$$

is semisimple. The semisimplicity of the Galois representations of function fields arising from abelian varieties was first proved by Zarhin $([\mathrm{Z}])$.

\section{Proof of the Theorem}

We first prove some lemmas.

Lemma 1. Let $G^{0}$ be a subgroup of $G$ with finite index. Let $G \rightarrow G L(V)$ be a finite dimensional representation of $G$. If the restriction $G^{0} \rightarrow G L(V)$ is semisimple, then $G \rightarrow G L(V)$ is also semisimple.

Proof. Let $U$ be a $G$-stable subspace of $V$. We need to show $U$ has a complement which is also $G$-stable. It is enough to show that there exists a homomorphism $P: V \rightarrow U$ such that $\left.P\right|_{U}$ is identity and $P$ is invariant under the action of $G$. Since $V$ is a semisimple representation of $G^{0}$, we can find a homomorphism $P_{0}$ 
such that $\left.P_{0}\right|_{U}$ is identity and that $P_{0}$ is $G^{0}$-invariant. Let $G^{0} g_{1}, \ldots, G^{0} g_{n}$ be representatives of the right cosets of $G^{0}$, where $n=\left[G: G^{0}\right]$. Define

$$
P=\frac{1}{n} \sum_{i=1}^{n} g_{i}^{-1} P_{0} g_{i}
$$

One can check $\left.P\right|_{U}$ is identity. To see $P$ is $G$-invariant, taking $g \in G$, then $G^{0} g_{1} g, \ldots, G^{0} g_{n} g$ are also representatives of the right cosets. So there is a permutation $\sigma$ of $\{1, \ldots, n\}$ such that $G^{0} g_{i} g=G^{0} g_{\sigma(i)}$. Hence there exists $g_{i}^{0} \in G^{0}$ such that $g_{i} g=g_{i}^{0} g_{\sigma(i)}$. We have

$$
\begin{gathered}
P g=\frac{1}{n} \sum g_{i}^{-1} P_{0} g_{i} g=\frac{1}{n} \sum g_{i}^{-1} P_{0} g_{i}^{0} g_{\sigma(i)} \\
=\frac{1}{n} \sum g_{i}^{-1} g_{i}^{0} P_{0} g_{\sigma(i)}=\frac{1}{n} \sum g g_{\sigma(i)}^{-1} P_{0} g_{\sigma(i)}=g P .
\end{gathered}
$$

So $P$ is $G$-invariant.

Lemma 2. Let $G^{0}$ be a normal subgroup of $G$. Assume there exists an exact sequence

$$
0 \rightarrow G^{0} \rightarrow G \stackrel{\operatorname{deg}}{\rightarrow} \mathbf{Z} \rightarrow 0
$$

We call the homomorphism $G \stackrel{\text { deg }}{\longrightarrow} \mathbf{Z}$ the degree homomorphism. Assume there exists an element in the center of $G$ with nonzero degree. Let $G \rightarrow G L(V)$ be a finite dimensional representation of $G$. If the restriction $G^{0} \rightarrow G L(V)$ is semisimple, and if there exists an element $g \in G$ with nonzero degree such that matrix corresponding to $g$ is diagonalizable, then $G \rightarrow G L(V)$ is semisimple.

Proof. Let $G_{d}=\{x \in G: d \mid \operatorname{deg}(x)\}$, where $d$ is the degree of an element in the center of $G$ with nonzero degree. Then $G_{d}$ is a subgroup of $G$ and $G / G_{d}$ is isomorphic to $\mathbf{Z} / d \mathbf{Z}$. By Lemma 1 , to prove $G \rightarrow G L(V)$ is semisimple, it is enough to prove the representation $G_{d} \rightarrow G L(V)$ is semisimple. Replacing $G$ by $G_{d}$ and $\operatorname{deg}$ by $\operatorname{deg} / d$, we may thus assume that there exists an elment in the center of $G$ with degree 1 ; that is, we may assume $G=G^{0} \times \mathbf{Z}$.

By assumption, the restriction of $G^{0} \times \mathbf{Z} \rightarrow G L(V)$ to $G^{0}$ is semisimple. So we have an isomorphism of $G^{0}$-representations:

$$
\begin{gathered}
\phi: \bigoplus_{j}\left(W_{j} \otimes \operatorname{Hom}_{G^{0}}\left(W_{j}, V\right)\right) \rightarrow V, \\
(w, f) \mapsto f(w),
\end{gathered}
$$

where the direct sum on the left-hand side sums over all the irreducible representations $W_{j}$ of $G^{0}$. If we let $n \in \mathbf{Z}$ act on $f \in \operatorname{Hom}_{G^{0}}\left(W_{j}, V\right)$ by $(n f)(w)=n f(w)$, where $n f(w)$ is the action of $n \in \mathbf{Z} \subset G^{0} \times \mathbf{Z}$ on $f(w) \in V$, then $\phi$ is also an isomorphism of $\left(G^{0} \times \mathbf{Z}\right)$-representations. So we may assume $V=\bigoplus_{j}\left(W_{j} \otimes U_{j}\right)$ as $\left(G^{0} \times \mathbf{Z}\right)$-representations, where $W_{j}$ are irreducible representations of $G^{0}$ and $U_{j}$ are some representations of $\mathbf{Z}$. By assumption, there exists an element $(g, n)$ with nonzero degree $n$ such that it corresponds to a diagonalizable matrix in $G L(V)$. So $(g, n)$ corresponds to a diagonalizable matrix in $G L\left(W_{j} \otimes U_{j}\right)$ for each $j$. Then $n$ corresponds to a diagonalizable matrix in $G L\left(U_{j}\right)$ because of the fact that if the tensor product of two matrices is diagonalizable, then each is. Hence $U_{j}$ is a semisimple representation of $\mathbf{Z}$. We can write $U_{j}=\bigoplus_{k} U_{j k}$, where each $U_{j k}$ is a 
one-dimensional representation of $Z$. We then have $V=\bigoplus_{j k}\left(W_{j} \otimes U_{j k}\right)$. Obviously each $W_{j} \otimes U_{j k}$ is an irreducible representation of $G^{0} \times \mathbf{Z}$. So $V$ is a semisimple representation of $G^{0} \times \mathbf{Z}$.

Now let's prove the theorem. I learned the following proof (and the above Lemma 2) from P. Deligne.

We use the same notation as in [D1]. The lisse $\overline{\mathbf{Q}_{l}}$-sheaf $\mathcal{F}_{0}$ gives rise to a $\overline{\mathbf{Q}}_{l^{-}}$ representation $\pi_{1}\left(X_{0}, \bar{x}\right) \rightarrow G L\left(\mathcal{F}_{\bar{x}}\right)$. Applying the construction [D1] 1.3.7 to $\mathcal{F}_{0}$, we get

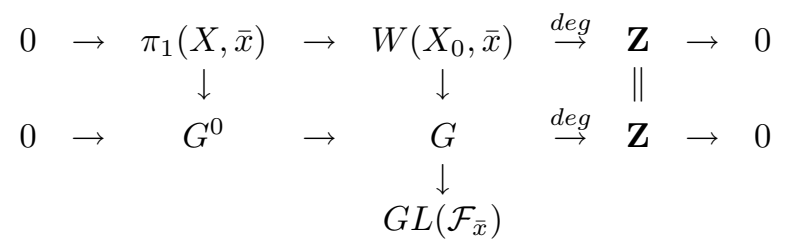

where $G^{0}$ is the Zariski closure of the image of $\pi_{1}(X, \bar{x}) \rightarrow G L\left(\mathcal{F}_{\bar{x}}\right)$, the group $W\left(X_{0}, \bar{x}\right)$ is the Weil group of $X_{0}$, and the second short exact sequence is obtained by pushing forward the first one using $\pi_{1}(X, \bar{x}) \rightarrow G^{0}$. By [D1] 3.4.1 (iii), $\mathcal{F}$ is a semisimple sheaf on $X$, that is, the representation $\pi_{1}(X, \bar{x}) \rightarrow G L\left(\mathcal{F}_{\bar{x}}\right)$ is semisimple. By [D1] 1.3.9, the algebraic group $G^{0}$ is an extension of a finite group by a semi-simple group, and by [D1] 1.3.11, if $Z$ is the center of $G$, then the restriction of the degree map on $Z$ has finite kernel and cokernel. ([D1] 1.3 .9 and 1.3.11 hold if one just assumes $\mathcal{F}$ is semisimple, as Deligne mentions in the proof of 1.3.9.) In particular, there exists an element in the center of $G$ with nonzero degree.

Now assume the Frobenius automorphism $F_{x}$ acts semisimply on $\mathcal{F}_{\bar{x}}$, and let's prove the representation $\pi_{1}\left(X_{0}, \bar{x}\right) \rightarrow G L\left(\mathcal{F}_{\bar{x}}\right)$ is semisimple. It is not hard to see the following statements are equivalent:

(1) $\pi_{1}\left(X_{0}, \bar{x}\right) \rightarrow G L\left(\mathcal{F}_{\bar{x}}\right)$ is semisimple.

(2) $W\left(X_{0}, \bar{x}\right) \rightarrow G L\left(\mathcal{F}_{\bar{x}}\right)$ is semisimple.

(3) $G \rightarrow G L\left(\mathcal{F}_{\bar{x}}\right)$ is semisimple.

We will prove (3) is true. By [D1] 3.4 .1 (iii), we know $\pi_{1}(X, \bar{x}) \rightarrow G L\left(\mathcal{F}_{\bar{x}}\right)$ is semisimple. It is not hard to see this implies that $G^{0} \rightarrow G L\left(\mathcal{F}_{\bar{x}}\right)$ is semisimple. By assumption, $F_{x}$ acts semisimply on $\mathcal{F}_{\bar{x}}$. Moreover there exists an element in the center of $G$ with nonzero degree, and the degree of $F_{x}$ is nonzero. So (3) holds by Lemma 2. This proves the theorem.

\section{ACKNOWLEDGEMENTS}

I thank Professor Deligne for suggesting Lemma 2 which greatly simplifies the proof of the theorem. This paper is written during my visit at Indiana University. I thank Professor Dadok for inviting me and for many interesting discussions on group theory.

\section{REFERENCES}

[D1] P. Deligne, La Conjecture de Weil, II, Publ. Math. IHES 52 (1980), 137-252. MR 83c:14017

[D2] P. Deligne, La Conjecture de Weil pour les Surfaces K3, Inventiones Math. 15 (1972), 206226. MR 45:5137

[PS] I. I. Piatetski-Shapiro and I. R. Shafarevich, The Arithmetic of K3 surfaces, Proc. Steklov Inst. Math. 132 (1975), 45-57. MR 49:302 
[T] J. Tate, Algebraic Cycles and the Poles of Zeta Functions, Arithmetic Algebraic Geometry, Harper and Row, New York (1965), 93-110. MR 37: 1371

[W] A. Weil, Courbes Algébriques et Variétés Abéliennes, Hermann, Paris (1971).

[Z] Y. Zarhin, Endomorphisms of Abelian Varieties over Fields of Finite Characteristic, Izv. Akad. Nauk SSSR Ser. Mat. 39 (1975), No. 2, 272-277. MR 51:8114

Institute of Mathematics, Nankai University, Tianjin, People's Republic of China

E-mail address: leifu@sun.nankai.edu.cn 